Original research article

\title{
Changes in the phenolic acids composition during pancake preparation: Whole and refined grain flour and processed food classification by UV and NIR spectral fingerprinting method-Proof of concept
}

\author{
Yingjian Lu ${ }^{\mathrm{a}}$, Ayaz Memon ${ }^{\mathrm{a}}$, Patrick Fuerst ${ }^{\mathrm{b}, \mathrm{c}}$, Alecia Kizonas $^{\mathrm{b}}$, Craig Morris ${ }^{\mathrm{c}}$, \\ Devanand Luthria ${ }^{\mathrm{a}, *}$ \\ a Beltsville Human Nutrition Research Center, Agricultural Research Service, U.S. Department of Agriculture, Beltsville, MD 20705, United States \\ ${ }^{\mathrm{b}}$ Department of Crop and Soil Sciences, Washington State University, Pullman, WA, 99164, United States \\ c Western Wheat Quality Lab., Agricultural Research Service, U.S. Department of Agriculture, Pullman, WA, 99164, United States
}

\section{A R T I C L E I N F O}

\section{Article history:}

Received 26 September 2016

Received in revised form 24 February 2017

Accepted 1 March 2017

Available online 4 March 2017

\section{Keywords:}

Food analysis

Wheat (Triticum)

Pancake preparation

Phenolic acids

Spectral fingerprinting

PCA

Two wheat cultivars

Whole and refined grain

Flour to batter to pancake

Proof of concept

\section{A B S T R A C T}

We investigated the changes in the levels of phenolic acids during pancake preparation (flour to batterpancake) from two refined and whole-wheat wheat varieties. In addition, we evaluated if multivariate analysis of ultraviolet (UV) and near infrared (NIR) spectral fingerprinting data can be used for classification of samples based on preparation stage, wheat varieties, and flour types (whole and refined). Results indicated that total phenolic acids did not significantly change $(<10 \%)$ during preparation of pancake from the refined and whole wheat flours. Most ( $>90 \%)$ of the phenolic acids existed in insoluble bound form and ferulic acid (82-92\%) was the most abundant phenolic acid. Correlation between simple UV spectral scan and HPLC analysis for the assay of phenolic acids ranged from 0.771 to 1.00. Principle component analysis (PCA) of NIR spectral data from 4900 to $5900 \mathrm{~cm}^{-1}$ showed clear separation between flour, batter, and pancake. Additionally, the PCA of UV spectral data between 250 and $350 \mathrm{~nm}$ separated two into clusters well (refined and whole-wheat samples). The results presented in this manuscript with limited number of samples illustrate the proof of concept that spectral fingerprinting techniques show promising potential for whole and refined grains sample classification and their products.

(c) 2017 Published by Elsevier Inc.

\section{Introduction}

Batter-based wheat products such as cakes, ice cream cones, pancakes, and waffles are consumed globally and are important sources of energy, carbohydrates, and other bioactive phytochemicals. A batter is made by mixing dry flours with ingredients such as water, milk, or eggs to the point that a pourable viscous flow is obtained. In the United States, pancakes or griddle cakes are commonly served at breakfast. Pancakes provide an effective model of batter-based products because its batter is fried or cooked on a hot griddle, this allows us to investigate the influence of processing on bioactive phytochemical compositions (Morris and Rose, 1996).

\footnotetext{
* Corresponding author.

E-mail address: Dave.Luthria@ars.usda.gov (D. Luthria).
}

Phenolic acids are widely distributed in grains and are present in high concentrations in whole-wheat products. Ferulic acid is the primary and most abundant phenolic acid in wheat grain. Smaller concentrations of caffeic, $p$-coumaric, sinapic acid, and other phenolic acids are also observed in wheat (Verma et al., 2009). Phenolic acids are present in comparatively higher concentrations in the germ and bran fractions as compared to the endosperm ( $\mathrm{Lu}$ et al., 2014a). They are thought to be one of the important groups of compounds responsible for health promoting properties of whole wheat products (Slavin et al., 2000; Truswell, 2002).

There are number of research investigation on nutrients on whole grain foods due to health benefits associated with the consumption of whole grains (Vitaglione et al., 2015). Dietary guidelines for Americans suggests at least half the grain consumed is whole grain (USDA, 2010). There are large number of studies showing the phytochemical content of whole grain is significantly higher as compared to the refined grain. Recently, Geng et al. (2015) showed the application of fuzzy chromatography mass 
chromatographic followed by chemometrics analysis (principal component analysis (PCA) and soft independent modeling of class analogy (SIMCA)) protocols for differentiation of whole and refined grains.

There have been a number of recent studies evaluating the effect of thermal treatments (cooking, frying, and baking) on the concentration of bioactive phytochemicals in the wheat-based products. Abdel-Aal and Rabalski (2013) found that baking increased the free phenolic acid while bound phenolic acids decreased or changed slightly in three whole grains cookies, muffins, and bread. In another study, baking decreased the content of total ester-bound phenolic acids and ferulic acid dehydrodimers in whole rye bread (Hansen et al., 2002). Additionally, Lu et al. (2014b) indicated that the total quantified phenolic acids did not change significantly when breads were prepared from refined and whole wheat flour. In another study by Lu et al. (2015) the authors pointed out that phenolic acids were relatively stable during baking, whereas tocopherols $(\sim 25-80 \%)$ and carotenoids ( $\sim 20-$ $80 \%$ ), were partially degraded. Furthermore, Kiszonas et al. (2015) carried out a study to investigate changes in the total arabinoxylans (TAX) and water-extractable arabinoxylans (WEAX) content during the making of pancakes. The authors found moderate changes in AX content during processing and a positive association of WEAX levels with end-product pancake volume.

As per our knowledge, there is no study reported in the literature on the changes in phenolic acids content during pancake preparation from whole and refined grain. The objective of our study was to investigate the changes in the levels of phenolic acids content during pancake preparation. We also determined the correlation between rapid UV spectral absorbance and the detailed LC-MS analysis for the determination of TPA in wheat-based matrix. Furthermore, we evaluated the application of simple ultraviolet and near infrared spectral fingerprints as a proof of concept to classify flour, batter, and pancake samples made from two wheat varieties, 'Louise' (soft white spring wheat) and 'Westbred 936' (WB 936, hard red spring wheat) using two types of wheat flours, refined (RF) and whole wheat (WW).

\section{Materials and methods}

\subsection{Wheat samples}

Two wheat varieties (Louise - soft white spring and Westbred 936 - hard red spring), grown in 2009 were used in the present study. Pancakes were prepared from both the refined (RF) and whole-wheat (WW) flours for each wheat variety. Grain was tempered and milled to approximately 70\% extraction (refined flour) in a Bühler MLU-202 flour mill (Bühler, Uzwil, Switzerland) (AACCI 1988a, 1988b). Whole wheat flour was produced by reducing the particle size of bran with a pin mill (model 91, Blendtec, Orem, UT, USA) so that $\geq 70 \%$ passed through a $180 \mu \mathrm{m}$ sieve and then blending with the refined flour. All the flour samples were stored at $-20^{\circ} \mathrm{C}$ prior to use.

\subsection{Pancake sample preparation method}

The pancakes were prepared as described in our earlier publications (AACCI 2015; Kiszonas et al., 2015). In short, dry pancake mixture consisted of $175 \mathrm{~g}$ of flour, $17.0 \mathrm{~g}$ of granulated sucrose, $5.5 \mathrm{~g}$ of dextrose, $3.2 \mathrm{~g}$ of sodium bicarbonate, $0.8 \mathrm{~g}$ of mono calcium phosphate, $3.5 \mathrm{~g}$ of sodium acid pyrophosphate, and $2.6 \mathrm{~g}$ of sodium chloride. Thus, the flour proportion in the dried batter and pancake was $80.1 \%$ of the total weight of dry ingredients plus oil. The leavening acids and sodium bicarbona25"/>g of dextrose, $3.2 \mathrm{~g}$ of sodium bicarbonate, $0.8 \mathrm{~g}$ of mono calcium phosphate, $3.5 \mathrm{~g}$ of sodium acid pyrophosphate, and $2.6 \mathrm{~g}$ of sodium chloride. Thus, the flour proportion in the dried batter and pancake was $80.1 \%$ of the total weight of dry ingredients plus oil. The leavening acids and sodium bicarbonate were kept separate from the other ingredients as well as from each other until combined with the other dry ingredients prior to use to prevent pre-reaction. The dry ingredients were mixed together at low speed with a Hobart N5 mixer from Hobart Corporation (Troy, OH, USA) or at medium speed with a KitchenAid 600 Series mixer from KitchenAid Corporation (St. Joseph, MI, USA) with a whisk attachment for $1 \mathrm{~min}$. Preparation of pancake is described in our earlier publication (Kiszonas et al., 2015). All analyses were performed on dried flour, batter, and pancake samples.

\subsection{Chemicals and reagents}

Phenolic acid (vanillic, caffeic, syringic, $p$-coumaric, ferulic, and sinapic acids) standards and ascorbic acid were purchased from Sigma-Aldrich (St. Louis, MO, USA). Sodium hydroxide and all solvents (ethyl ether, ethyl acetate, and methanol) were obtained from Fisher Chemicals (Fair Lawn, NJ, USA). All chemicals and solvents were either analytical grade or HPLC grade and were used directly without further purification. EDTA (ethylene diamine tetra acetic acid) was purchased from EMD Chemicals (Gibbstown, NJ, USA). Polyvinylidene difluoride (PVDF) syringe filters with pore size $0.45 \mu \mathrm{m}$ were purchased from National Scientific Company (Duluth, GA, USA).

\subsection{Phenolic acids analysis}

The ground flour, batter, or pancake sample $(250 \pm 1 \mathrm{mg})$ was separately extracted for soluble (free and conjugated) and insoluble bound phenolic acids using ultrasonic assisted extraction as described previously (Lu et al., 2015). After evaporating the organic phase under nitrogen, the residue was re-dissolved in methanol: water $(75: 25, \mathrm{v} / \mathrm{v})$ and filtered through a $0.45 \mu \mathrm{m}$ membrane filter. The filtered extract was used for the analysis of soluble (free and conjugated) and insoluble bound phenolic acids by LC-MS.

\subsection{Analysis of phenolic acids by HPLC-ESI-MS}

Quantification of phenolic acids from all samples was carried out using an Agilent 1290 Infinity LC system coupled to a diode array (DA) and a mass spectrometry (MS) detectors from Agilent Technologies (Palo Alto, CA, USA) as previously described (Luthria et al., 2012). Identification of phenolic acids was achieved by comparison of mass and ultraviolet spectral data as previously reported and by comparison of retention time with authentic commercial phenolic acids standard (Luthria et al., 2006). Briefly, separation of phenolic acids was achieved using a reversed phase C18 Luna column $(150 \times 4.6 \mathrm{~mm}$; particle size $5 \mu \mathrm{m})$ from Phenomenex (Torrance, CA, USA) at ambient temperature (between 22 and $27^{\circ} \mathrm{C}$ ). Separation was achieved using a binary gradient with two solvents. Solvent A was $0.1 \%(v / v)$ formic acid in water and solvent B was $0.1 \%(\mathrm{v} / \mathrm{v})$ formic acid in methanol. The flow rate was set to $1 \mathrm{~mL} / \mathrm{min}$ and $20 \mu \mathrm{L}$ of sample was used for injection. The linear gradient consisted of $5 \%$ (B) to $30 \%$ (B) for $25 \mathrm{~min}$, followed by $30 \%$ (B) for $35 \mathrm{~min}$, then ramped from $30 \%$ (B) to $100 \%$ (B) for $10 \mathrm{~min}$ and held at $100 \%$ (B) for $5 \mathrm{~min}$. The mobile phase concentration was returned to $5 \%$ (B) and held for $10 \mathrm{~min}$ before the next injection. The mass spectral data were collected in both positive and negative ion modes at low and high fragment voltages ( $70 \mathrm{~V}$ and $250 \mathrm{~V}$ ). The instrument was set to scan from 100 to 750 mass units. The temperature of the drying gas was $300^{\circ} \mathrm{C}$ at a flow rate of $12 \mathrm{~L} / \mathrm{min}$, capillary voltage for both positive and negative ion modes was adjusted at $3000 \mathrm{~V}$ and a nebulizer 
pressure of $345 \mathrm{kPa}$. The LC system was directly connected to a mass spectrometer with no stream splitting. All Samples were extracted and analyzed in four replicates.

\subsection{UV spectral fingerprinting}

Flour, batter, and pancake extracts from HPLC analysis of phenolic acids $(200 \mu \mathrm{L})$ were placed in a 96 well Costar plate with a UV transparent flat bottom (Corning Inc., NY, USA). The extracts were scanned from 250 and $500 \mathrm{~nm}$ in the Spectramax Plus 384 plate reader (Molecular Devices, CA, USA). Columns 1 and 12 in 96 well plates were filled with $\mathrm{MeOH}: \mathrm{H}_{2} \mathrm{O}(80: 20, \mathrm{v} / \mathrm{v}$, blank) and four replicates extraction and analysis was carried out with samples from different pancake preparation stages. The spectral absorbance data for each extract was converted into an Excel (Microsoft, Inc., Belleview, WA, USA) format for statistical analysis. Spectra were from 250 to $500 \mathrm{~nm}$ at $1 \mathrm{~nm}$ intervals. Each extract was analyzed in quadruplicate. The resultant data matrix, 48 samples by 251 absorption intensities were used for statistical analysis.

\subsection{NIR spectral fingerprinting}

Approximately $1 \mathrm{~g}$ of the dried powdered flour, batter, or pancake sample was placed in an $8 \mathrm{~mL}$ glass vial and tamped on a solid surface to ensure that the powder was settled firmly. The powdered samples were scanned between 4000 and $10,000 \mathrm{~cm}^{-1}$ at approximately $2 \mathrm{~cm}^{-1}$ intervals in the NIR spectrometer Nicolet 6700 (Thermo-Electron, Waltham, MA, USA). Each sample was analyzed six times after remixing the samples be shaking resulting in 72 spectral fingerprints. Spectra were exported from the NIR spectrometer to Excel (Microsoft, Inc., Belleview, WA, USA). The resultant data matrix, was further analyzed using standalone chemometrics software: Solo (Eigenvector Research, Inc., Manson, WA, USA). Preprocessing in Solo consisted of normalization (normalized to unit vector) and mean centering.

\subsection{Statistical analysis}

Data were reported as means \pm standard deviations (SD) for four replicates treatments. One-way analysis of variance (ANOVA) and Tukey's tests were performed using SPSS (SPSS for Windows, version 10.0.5, SPSS Inc., Chicago, IL, USA). Correlation analyses were performed using a two-tailed Pearson's correlation test. Statistical significance was declared at $P<0.05$.

\section{Results and discussion}

3.1. Comparison of phenolic acids in refined vs. whole wheat samples from different pancake preparation stages

Six point calibration curves for the four commercial phenolic acids identified in all samples were constructed using diode-array detection method. The linear regression equation and the correlation coefficient for each identified phenolic acid was determined (caffeic acid - Eq $\mathrm{y}=1896.4 \mathrm{x}-47.617, \mathrm{R}^{2}$ 0.9999; $p$ coumaric acid - Eq y =499.31x +5.419, $\mathrm{R}^{2} 0.9969$; ferulic acid Eq $y=2680.3 x+16.041, \quad R^{2} \quad 0.9995 ;$ sinapic acid Eq $\left.y=1917.3 x-3.165, R^{2} 0.9997\right)$. The limit of detection and the limit of quantification for phenolic acids was determined as $0.05 \mu \mathrm{g} / \mathrm{mL}$ and $0.2 \mu \mathrm{g} / \mathrm{mL}$ respectively. The quantities of total phenolic acids extracted from the RF flour samples were significantly lower than those from the WW flour samples (Table 1). This is in agreement with the previously published reports from our lab and other authors where only about one tenth of the total phenolic acids content in RF wheat flour was observed as compared to the WW flour (Beta et al., 2005; Lu et al., 2015; Mattila et al., 2005).

The phenolic acid composition and content in RF and WW flour, batter, and pancake are presented in Table 1. In terms of overall abundance, ferulic acid (FA) was the most abundant phenolic acid, accounting for a mean of $85 \%$ (ranged from 84 to $87 \%$ ) of TPA among RF samples and a mean of 90\% (ranged from 89 to 90\%) among WW samples. Sinapic acid was second most abundant phenolic acid, accounting for a mean of $9 \%$ and $6 \%$ of TPA in RF and WW samples, respectively. Among all samples evaluated, TPA ranged from 10.2- to 12.9-fold greater in WW samples than in RF samples (Table 1). All individual phenolic acids were also higher in WW than in RF samples (Table 1). The greatest increases came from FA, which had at most a 13.2-fold increase, in WW compared to RF flour when averaged across all samples in different pancake stages and varieties. Our results are consistent with previous studies demonstrating that total phenolic acids were around 10fold greater in whole wheat than in refined wheat products (Mattila et al., 2005).

The huge increase in FA in WW compared to RF samples correlated well with previous study where higher level (8-15-fold) of FA was detected in bran than in RF flour samples (Andreasen et al., 2000). Ferulates were also far more abundant (about 100fold) in outer layer of the wheat kernel, including bran and germ, compared to starchy endosperm tissue (Barron et al., 2007).

Table 1

Individual and total phenolic acids (TPA) of the flour, batter, and pancake prepared from Louise (soft white wheat) and WB936 (hard red wheat) variety*.

\begin{tabular}{|c|c|c|c|c|c|c|}
\hline Type & Preparation stage & $\mathrm{CA}(\mu \mathrm{g} / \mathrm{g})$ & $p-C A(\mu g / g)$ & $\mathrm{FA}(\mu \mathrm{g} / \mathrm{g})$ & $\mathrm{SiA}(\mu \mathrm{g} / \mathrm{g})$ & TPAs $(\mu \mathrm{g} / \mathrm{g})$ \\
\hline Louise RF & Flour & $2.17 \pm 0.04 a$ & $1.70 \pm 0.07 a$ & $48.75 \pm 1.70 \mathrm{a}$ & $3.50 \pm 0.06 a$ & $56.13 \pm 2.00 a$ \\
\hline Louise RF & Batter & $2.63 \pm 0.09 a$ & $2.29 \pm 0.12 \mathrm{a}$ & $48.45 \pm 2.43 a$ & $4.07 \pm 0.45 a$ & $57.43 \pm 0.85 a$ \\
\hline Louise RF & Pancake & $2.72 \pm 0.13 a$ & $2.40 \pm 0.22 a$ & $50.81 \pm 1.06 a$ & $4.50 \pm 0.35 a$ & $60.44 \pm 1.21 a$ \\
\hline Louise WW & Flour & $12.12 \pm 1.72 b c$ & $24.67 \pm 1.01 \mathrm{c}$ & $657.13 \pm 19.14 b$ & $58.19 \pm 13.70 \mathrm{~d}$ & $752.11 \pm 22.31 b c$ \\
\hline Louise WW & Batter & $11.15 \pm 1.17 \mathrm{~b}$ & $24.07 \pm 1.94 \mathrm{c}$ & $670.76 \pm 22.57 b$ & $33.01 \pm 1.92 b$ & $738.98 \pm 23.63 b$ \\
\hline Louise WW & Pancake & $13.18 \pm 1.78 b c$ & $24.67 \pm 1.21 \mathrm{c}$ & $683.52 \pm 37.14 b$ & $51.34 \pm 16.74 \mathrm{~cd}$ & $772.71 \pm 27.64 c$ \\
\hline WB936 RF & Flour & $2.45 \pm 0.10 a$ & $2.46 \pm 0.20 \mathrm{a}$ & $59.93 \pm 1.64 a$ & $6.94 \pm 2.57 a$ & $71.78 \pm 2.23 a$ \\
\hline WB936 RF & Batter & $2.78 \pm 0.10 \mathrm{a}$ & $2.18 \pm 0.27 a$ & $51.67 \pm 3.08 \mathrm{a}$ & $4.27 \pm 1.72 \mathrm{a}$ & $60.90 \pm 2.80 \mathrm{a}$ \\
\hline WB936 RF & Pancake & $3.09 \pm 0.13 a$ & $2.49 \pm 0.14 a$ & $60.61 \pm 5.09 a$ & $7.30 \pm 2.08 a$ & $73.49 \pm 3.95 a$ \\
\hline WB936 WW & Flour & $13.69 \pm 0.90 c$ & $15.62 \pm 0.61 b$ & $714.31 \pm 15.51 c$ & $64.94 \pm 4.16 \mathrm{~d}$ & $808.57 \pm 11.49 d$ \\
\hline WB936 WW & Batter & $12.21 \pm 2.18 b c$ & $15.77 \pm 0.36 b$ & $763.72 \pm 16.28 c$ & $40.16 \pm 2.16 b c$ & $831.85 \pm 16.66 d$ \\
\hline WB936 WW & Pancake & $16.37 \pm 1.17 d$ & $15.05 \pm 0.76 b$ & $756.46 \pm 7.34 c$ & $54.43 \pm 4.42 \mathrm{~d}$ & $842.31 \pm 9.90 d$ \\
\hline
\end{tabular}

Note: RF, refined; WW, whole wheat; CA, caffeic acid; $p$-CA, p-coumaric acid; FA, ferulic acid; SiA, sinapic acid; TPAs, total phenolic acids. The results were expressed as Mean \pm SD of four replicate extraction and analysis $(n=4)$ followed by a letter. Same letter means no statistical difference whereas different letter stands for significant statistical difference $(P>0.05)$. 
3.2. Significance of processing on phenolic acids during pancake preparation

TPA and FA contents in pancakes (60.44 and $73.49 \mu \mathrm{g} / \mathrm{g}$ dry weight (DW)) were similar or marginally higher than the batter and flour in the refined samples for both wheat varieties (Table 1). The pancake made from refined flours also had slightly higher amounts of caffeic, $p$-coumaric, ferulic, and TPA in both Louise and WB936 wheat varieties. Similarly, TPA and FA trends among WW (flour, batter, and pancake) samples were observed. The WW pancake showed similar or marginal increase in most individual phenolic acids and TPA content than the corresponding flour and batter samples. The above results indicate that thermal processing did not reduce the levels of phenolic acids in prepared pancake samples.

\subsection{Comparison of phenolic acid content in two wheat varieties}

When comparing RF flour samples for the two varieties, caffeic, ferulic, sinapic, and TPA content were always higher in WB936 than in Louise (Table 1). In the WW samples, caffeic, ferulic, sinapic, and total phenolic acids contents in WB936 were consistently higher as expected, but the levels of $p$-coumaric acid was greater in Louise than in WB936 wheat variety. In WW pancake sample, FA and TPA content was significantly higher in WB936 (756.46 $\mu \mathrm{g} / \mathrm{g} \mathrm{DW}$; $842.31 \mu \mathrm{g} / \mathrm{g}$ DW) than in Louise $(683.52 \mu \mathrm{g} / \mathrm{g}$ DW; $772.71 \mu \mathrm{g} / \mathrm{g}$ DW) (Table 1). These were in agreement with previously reported results (Whent et al., 2012).

\subsection{NIR and UV spectral fingerprints}

Fig. 1A shows the UV spectral fingerprints $(250-350 \mathrm{~nm})$ and Fig. 1B depicts near infrared spectral fingerprints (4900$5900 \mathrm{~cm}^{-1}$ ) of the flour, batter, and pancake made from two refined and whole wheat cultivars. For UV analysis each sample was extracted four times and analyzed and for NIR analysis each sample was analyzed in six replicates. The relative standard
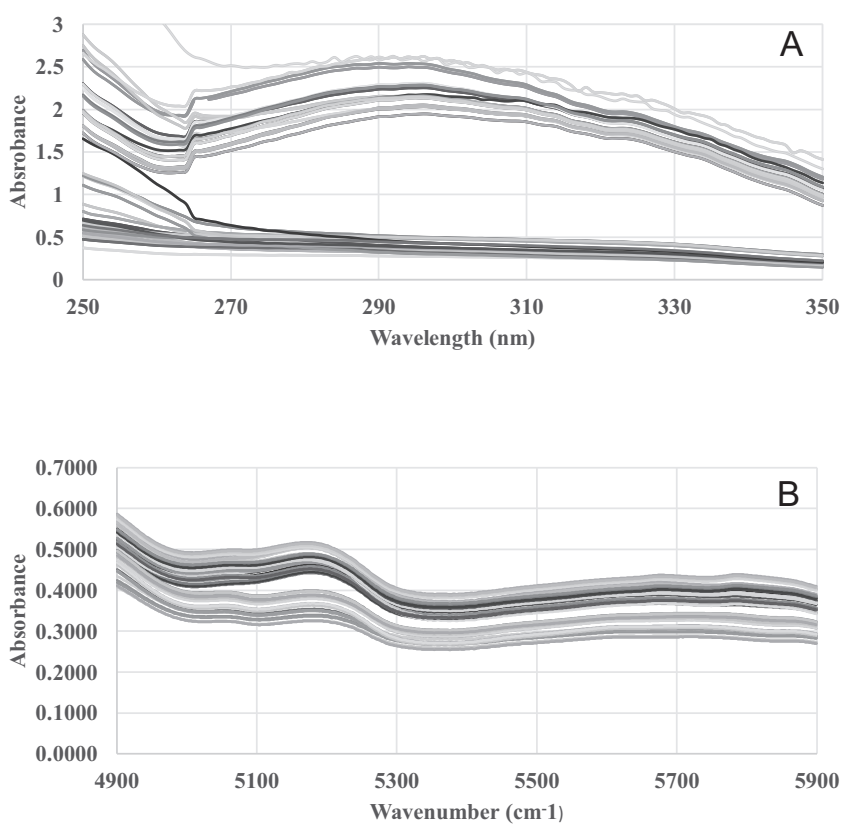

Fig. 1. A. UV spectral fingerprints $(250-350 \mathrm{~nm})$ and B. Near infrared spectral fingerprints $\left(4900-5900 \mathrm{~cm}^{-1}\right)$ of the flour, batter, and pancake made from two refined and whole wheat cultivars. For UV analysis each sample was extracted four times $(n=4)$ and analyzed and for NIR analysis each sample was analyzed in six replicates $(n=6)$. deviation was less than $5 \%$ for all UV and NIR spectral fingerprint at three different wavelengths or wavenumber. The UV and NIR spectral fingerprints Spectral fingerprinting is an un-targeted method used for classification of samples based on spectral patterns without identification or quantification of specific components. NIR spectral fingerprints are obtained on powdered solid material and UV spectral fingerprints were acquired in MeOH: $\mathrm{H}_{2} \mathrm{O}(80: 20, v / v)$ extract of fraction obtained after base hydrolysis. Pattern recognition for the NIR and UV spectral data for the classification was achieved used unsupervised PCA. The first and the second derivative plots of the spectral data showed that maximum variation in the UV spectral data was between 250 and $350 \mathrm{~nm}$ and $4900-5900 \mathrm{~cm}^{-1}$ for the near infrared spectral data. Therefore these regions were selected for classification of sample using PCA.

The PCA score plots for NIR spectral data between 4900 and $5900 \mathrm{~cm}^{-1}$ is shown in Fig. 2. The abbreviations used in the score plot for sample identification are described in Table 2. The score plot with limited number of samples showed distinct separation as a proof of concept between the 12 different sample sets. Over $99 \%$ of the variance was captured by PC1, PC2, and PC3. The two wheat cultivars, Louise and WB936 were well separated along component 3. All flour samples were located on the left side in the negative PC1 area, and were separated from the processed samples, batter and pancakes located on the right side in the positive region of PC1. In both wheat cultivars, refined and whole, flour, batter, and pancake samples were again grouped together. Thus simple principal component analysis of the NIR spectral data showed significant potential for analysis refined and whole wheat processed product prepared from different wheat cultivars. Similar separation of whole and refined wheat samples was recently reported by Geng et al. (2015) using fuzzy chromatography mass chromatographic using multivariate analysis tools namely, PCA and SIMCA. In addition, Fig. 2 also showed that the score plots might separate well between flour types (RF based vs. WW based).

The PCA score plots of the UV spectral data analysis showed distinct separation between all refined and whole-wheat samples (Fig. 3). All whole-wheat samples were located on the left lower side in the negative PC1 area, and all refined samples were on the opposite side (positive PC1). This can be attributed to the phenolic acid content which show strong absorption between 250 and $350 \mathrm{~nm}$ (Robbins, 2003). In addition, the results presented in Table 2 on phenolic acid analysis shows over 10-folds greater TPA content in WW samples as compared to RF samples (Table 1) as reported in the present and earlier reported studies (Mattila et al., 2005). Recently, Martelo-Vidal and Vazquez (2014) used similar UV-VIS-NIR spectroscopy method to analyze wine based on phenolic content. The distinction between flour, batter, and pancake within each subgroup (whole or refined) was not clear as compared to the NIR data. Thus simple UV spectral studies showed similar classification between whole and refined wheat sample as observed in recently published study (Geng et al., 2015).

\subsection{Correlations between UV spectral absorbance and HPLC analysis for phenolic acids}

Simple UV spectral methodology provides rapid assay of TPA. This method was used for estimation of chlorogenic acid in eggplants (Luthria, 2012). According to Robbins (2003), the optimal spectral absorbance for phenolic acids (hydroxybenzoic acid and hydroxycinnamic acid derivatives) range between 270 and $360 \mathrm{~nm}$. In the present study, we compared that application of simplified spectral method with detailed LC-MS analysis for the estimation of TPA. Pearson's correlation coefficients between UV spectral absorbance $\left(\lambda_{\max } 280 \mathrm{~nm}\right.$ and $310 \mathrm{~nm}$ ) and detailed HPLC analysis of individual and total phenolic acids are shown in Table 3. 


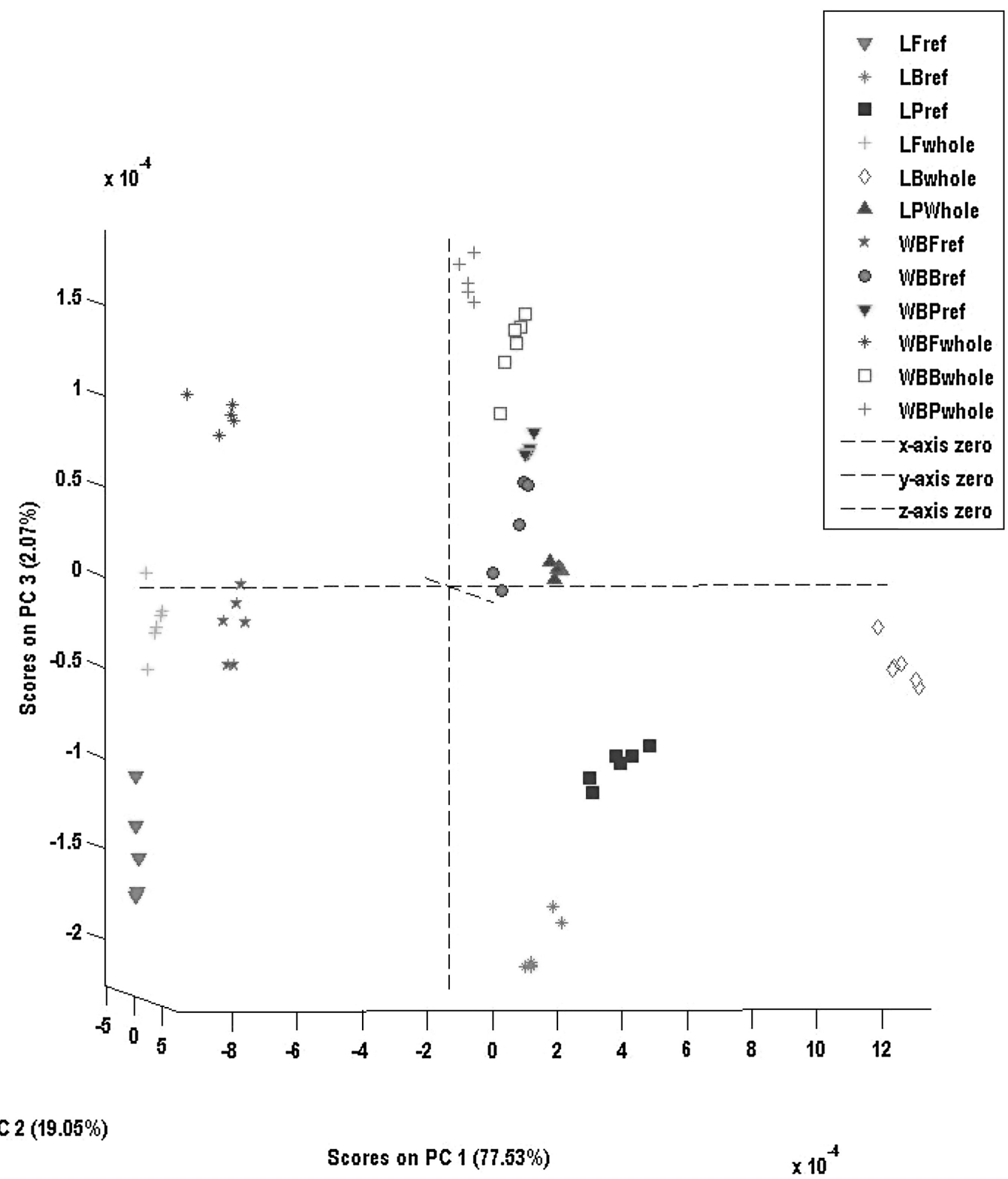

Fig. 2. Principal component analysis of Near Infrared spectral fingerprints of flour, batter, and pancake made from two refined and whole-wheat wheat varieties. Each sample was analyzed in six replicate $(n=6)$. Abbreviations: L: Louise wheat cultivar; W: Westbred 936 wheat cultivar; ref: refined wheat; whole: whole wheat; F: Flour; B: Batter: P: Pancake.

Table 2

Sample ID for 12 wheat samples.

\begin{tabular}{llll}
\hline Sample ID & Wheat cultivar & Wheat Fraction & Flour Type \\
\hline LFref & Louise & Flour & Refined Wheat Flour \\
LBref & Louise & Batter & Refined Wheat Flour \\
LPref & Louise & Pancake & Refined Wheat Flour \\
LFwhole & Louise & Flour & Whole Wheat Flour \\
LBwhole & Louise & Batter & Whole Wheat Flour \\
LPwhole & Louise & Pancake & Whole Wheat Flour \\
WBFref & Westbred 936 & Flour & Refined Wheat Flour \\
WBBref & Westbred 936 & Batter & Refined Wheat Flour \\
WBPref & Westbred 936 & Pancake & Refined Wheat Flour \\
WBFwhole & Westbred 936 & Flour & Whole Wheat Flour \\
WBBwhole & Westbred 936 & Batter & Whole Wheat Flour \\
WBPwhole & Westbred 936 & Pancake & Whole Wheat Flour \\
\hline
\end{tabular}

UV spectral data showed significantly strong correlations $(r$ $\geq 0.970$ ) between UV spectral data and ferulic acid (predominant phenolic acid) content as determined by LC-MS analysis in different flour, batter, and pancake samples. These results suggest that a simple UV spectral method can be used to estimate the concentration of phenolic acids in base hydrolyzed flour, batter, and pancake extracts. However, this positive correlation cannot be directly extended or used for the assay of other phenolic acids in different matrices as other phytochemicals may have absorbance coefficient in this region and may potentially interfere with direct quantification.

\section{Conclusions}

The results presented in the current study showed that the concentration and distribution of phenolic acids varied with type of wheat flours (whole and refined) and cultivars (Louise - soft 


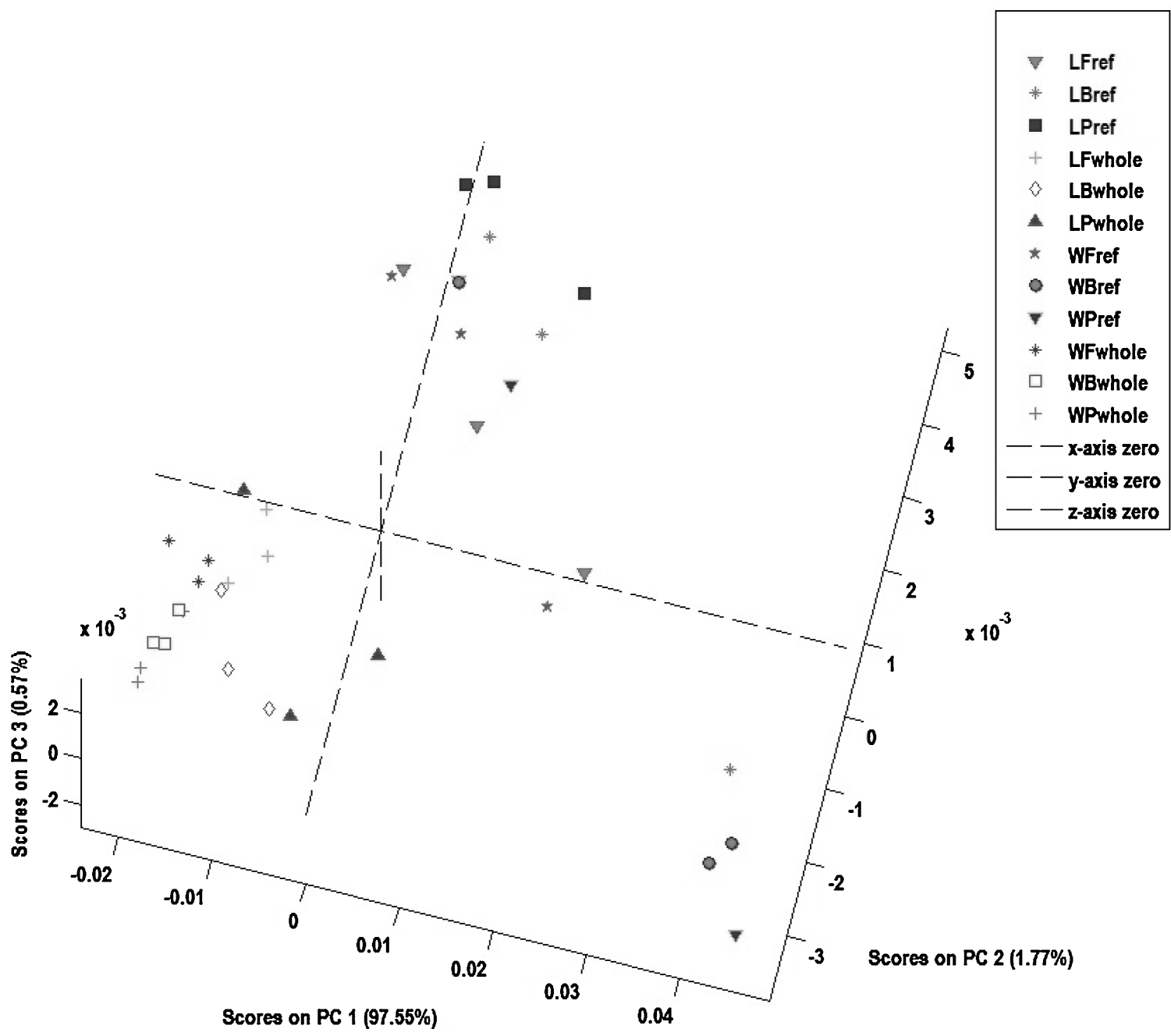

Fig. 3. Principal component analysis of ultraviolet spectral fingerprints of flour, batter, and pancake extracts made from two refined and whole-wheat wheat varieties. Each sample was extracted and analyzed in four replicates $(n=4)$. Abbreviations: L: Louise wheat cultivar; W: Westbred 936 wheat cultivar; ref: refined wheat; whole: whole wheat; F: Flour; B: Batter: P: Pancake.

Table 3

Correlation between UV spectroscopy and HPLC analysis methods for the assay of phenolic acids.

\begin{tabular}{lllllll}
\hline & $280 \mathrm{~nm}$ & $310 \mathrm{~nm}$ & CA & $p$-CA & FA & SiA \\
\hline 310 nm & $0.998^{* *}$ & & & & & \\
CA & $0.952^{* *}$ & $0.968^{* *}$ & & & & \\
p-CA & $0.965^{* *}$ & $0.956^{* *}$ & $0.885^{* *}$ & & & \\
FA & $0.970^{* *}$ & $0.980^{* *}$ & $0.985^{* *}$ & $0.915^{* *}$ & & \\
SiA & $0.871^{* *}$ & $0.891^{* *}$ & $0.953^{* *}$ & $0.771^{* *}$ & $0.928^{* *}$ & \\
TPA & $0.970^{* *}$ & $0.981^{* *}$ & $0.987^{* *}$ & $0.915^{* *}$ & $1.000^{* *}$ & $0.933^{* *}$ \\
\hline
\end{tabular}

Results without asterisks were not significant at $p<0.05 .280 \mathrm{~nm}$, UV spectral at $280 \mathrm{~nm} ; 310 \mathrm{~nm}$, UV spectral at $310 \mathrm{~nm}$; CA, Caffeic acid; $p$-CA, $p$-Coumaric acid; FA, Ferulic acid; SiA - Sinapic acid; TPA - Total phenolic acids; The results were expressed as Pearson correlation coefficients ( $r$ value). ${ }^{*} p<0.05$.

*** $p<0.01$

white spring and Westbred 936 - hard red spring). Concentrations of phenolic acids were higher in all of the whole wheat (WW) samples than in the refined (RF) wheat samples. Westbred 936 had higher phenolic acid concentrations as compared to Louise cultivar. PCA of NIR and UV spectral data with limited number of samples provides the proof of concept that spectral fingerprinting techniques shows promising potential for sample classification. Multivariate analysis of the NIR spectral fingerprints separated flour, batter, and pancake samples made from refined and whole wheat. PCA of UV spectral data classified refined and whole-wheat samples due to higher concentration of phenolic acid present in the bran part of the whole wheat grain. However, flour, batter, and pancake fractions within each subgroup (whole and refined) were not well separated. In addition, a simple UV spectral scan can differentiate whole and refined grain products based on simple absorption scan between 260 and $330 \mathrm{~nm}$. Significant correlation of individual and total phenolic acids between UV spectral scan method and HPLC analysis was observed.

\section{Acknowledgements}

The authors wish to acknowledge Mrs. Samina Shami of USDAARS for her assistance in carrying out experimentation. This project was supported by the Agriculture and Food Research Initiative Grant no. 2009-02347 from the USDA National Institute of Food and Agriculture.

\section{References}

American Association of Cereal Chemists International - AACCI, 1988a. Approved Methods 26-21.02. Experimental Milling-Bühler Method for Hard Wheat. AACC International, St. Paul, MN (Approved October 12).

American Association of Cereal Chemists International - AACCI, 1988b. Approved Methods 26-31.01. Experimental Milling-Bühler Method for Soft Wheat 
Straight-Grade Flour. AACC International, St. Paul, MN Approved October 12, Available online only.

American Association of Cereal Chemists International - AACCI, 2015. Approved Methods 10-80.01. Flour Quality in a Pancake-Making Method, 11th ed. The Association, St. Paul, MN (Approved December).

Abdel-Aal, E.S.M., Rabalski, I., 2013. Effect of baking on free and bound phenolic acids in whole grain bakery products. J. Cereal Sci. 57, 312-318.

Andreasen, M.F., Christensen, L.P., Meyer, A.S., Hansen, Å., 2000. Ferulic acid dehydrodimers in rye (Secale cereal L.). J. Cereal Sci. 31, 303-307.

Barron, C., Surget, A., Rouau, X., 2007. Relative amounts of tissue in mature wheat (Triticum aestivum L.) grain and their carbohydrate and phenolic acid composition. J. Cereal Sci. 45, 88-96.

Beta, T., Shin, N., Dexter, J.E., Sapirstein, H.D., 2005. Phenolic content and antioxidant activity of pearled wheat and roller-milled fractions. Cereal Chem. 82, 390-393.

Geng, P., Zhang, M., Harnly, J.M., Luthria, D.L., Chen, P., 2015. Use of fuzzy chromatography mass spectrometric (FCMS) fingerprinting and chemometrics analysis for differentiation of whole-grain and refined wheat (T. aestivum) flour. Anal. Bioanal. Chem. 407, 7875-7888.

Hansen, H.B., Andresen, M.F., Nielsen, M.M., Larsen, L.M., Knudsen, K.E.B., Meyer A S., Christensen, L.P., Hansen, A., 2002. Changes in dietary fiber, phenolic acids and activity of endogenous enzymes during rye bread-making. J. Eur. Food Res. Technol. 214, 33-42.

Kiszonas, A.M., Fuerst, E.P., Luthria, D., Morris, C.F., 2015. Tracking arabinoxylans through the preparation of pancakes. Cereal Chem. 92, 37-43.

Lu, Y., Lv, J., Yu, L., Bayard, A., Costa, J., Yu, L., Luthria, D., 2014a. Phytochemical compositions and antiproliferative activities of bran fraction of ten Marylandgrown soft winter wheat cultivars: comparison of different radical scavenging assays. J. Food Compos. Anal. 36, 51-58.

Lu, Y., Luthria, D., Fuerst, E.P., Kiszonas, A.M., Yu, L., Morris, C.F., 2014b. Effect of processing on phenolic composition of dough and bread fractions made from refined and whole wheat flour of three wheat varieties. J. Agric. Food. Chem. 62, 10431-10436.

Lu, Y., Fuerst, E.P., Lv, J., Morris, C.F., Yu, L., Fletcher, A., Kiszonas, A.M., Yu, L., Luthria D., 2015. Phytochemical profile and antiproliferative activity of dough and bread fractions made from refined and whole wheat flours. Cereal Chem. 92, 271-277.

Luthria, D.L., Mukhopadhyay, S., Krizek, D.T., 2006. Content of total phenolics and phenolic acids in tomato (Lycopersicon esculentum Mill.) fruits as influenced by cultivar and solar UV radiation. J. Food Compos. Anal. 19, 771-777.
Luthria, D.L., Liu, K., Memon, A.A., 2012. Phenolic acids and antioxidant capacity of distillers dried grains with solubles (DDGS) as compared with corn. J. Am. Oil Chem. Soc. 89, 1297-1304

Luthria, D.L., 2012. A simplified 96-well method for the estimation of phenolic acids and antioxidant capacity from eggplant pulp extracts using UV spectral scan data. J. Funct. Foods 4, 238-242.

Martelo-Vidal, M.J., Vazquez, M., 2014. Determination of polyphenolic compounds of red wines by UV-VIS-NIR spectroscopy and chemometrics tools. Food Chem. 158, 28-34.

Mattila, P., Pihlava, J., Hellström, J., 2005. Contents of phenolic acids, alkyl-and alkenylresorcinols, and avenanthramides in commercial grain products. J. Agric. Food. Chem. 53, 8290-8295.

Morris, C.F., Rose, S.P., 1996. Wheat - Cereal Grain Quality. In: Henry, R.J., Kettlewell, P.S. (Eds.), Chapman and Hall, U.K, pp. 3-54.

Robbins, R.J., 2003. Phenolic acids in foods: an overview of analytical methodology. J. Agric. Food. Chem. 51, 2866-2887.

Slavin, J., Marquart, L., Jacobs, D., 2000. Consumption of whole-grain foods and decreased risk of cancer: proposed mechanisms. Cereal Food World 45, 54-58.

Truswell, A.S., 2002. Cereal grains and coronary heart disease. Eur. J. Clin. Nutr. 56, $11-14$.

USDA, United States Department of Agriculture, 2010. Dietary Guidelines for Americans 2010. . http://www.cnpp.usda.gov/sites/default/files/ dietary_guidelines_for_americans/PolicyDoc.pdf.

Verma, B., Hucl, P., Chibbar, R.N., 2009. Phenolic acid composition and antioxidant capacity of acid and alkali hydrolyzed wheat bran fractions. Food Chem. 116 947-954.

Vitaglione, P., Mennella, I., Ferracane, R., Rivellese, A.A., Giacco, R., Ercolini, D. Gibbons, S.M., La Storia, A., Gilbert, J.A., Jonnalagadda, S., Thielecke, F., Gallo, M. A., Scalfi, L., Fogliano, V., 2015. Whole-grain wheat consumption reduces inflammation in a randomized controlled trial on overweight and obese subjects with unhealthy dietary and lifestyle behaviors: role of polyphenols bound to cereal dietary fiber. Am. J. Clin. Nutr. 101, 251-261.

Whent, M., Huang, H., Xie, Z., Lutterodt, H., Yu, L., Fuerst, E., Morris, C., Yu, L., Luthria, D., 2012. Phytochemical composition, anti-inflammatory, and antiproliferative activity of whole wheat flour. J. Agric. Food. Chem. 60, 2129-2135. 